\title{
Supporting Information: A Seven-Site Effective Pair Potential for Simulating Liquid Water
}

Chong-Li Zhao ${ }^{\dagger}$, Dong-Xia Zhao ${ }^{*,}$, Cui-Cui Bei ${ }^{\dagger}$, Xiang-Na Meng ${ }^{\dagger}$, Shenmin Li*, Zhong-Zhi Yang*,†

†School of Chemistry and Chemical Engineering, Liaoning Normal University, Dalian, 116029, People's Republic of China

ॠCollege of Environmental and Chemical Engineering, Dalian University, Dalian, 116622, People's Republic of China

Corresponding authors E-mail address:

zzyang@lnnu.edu.cn (Zhong-Zhi Yang)

zhaodxchem@Innu.edu.cn (Dong-Xia Zhao)

Tel: $+86411-82159607$

lishenmin@dlu.edu.cn (Shenmin Li)

Tel: +86 411-87403949 
Table S1. Density (in $\mathrm{g} / \mathrm{cm}^{3}$ ) calculated using TIP7P model over the temperatures between $253 \mathrm{~K}$ and $373 \mathrm{~K}$ at $1 \mathrm{~atm}$.

\begin{tabular}{lll}
\hline Temperature & TIP7P & Experiment \\
\hline 253.15 & $0.9946 \pm 0.0005$ & 0.9936 \\
258.15 & $0.9969 \pm 0.0003$ & 0.9963 \\
263.15 & $0.9986 \pm 0.0003$ & 0.9981 \\
268.15 & $0.9997 \pm 0.0002$ & 0.9993 \\
273.15 & $1.0004 \pm 0.0002$ & 0.9998 \\
277.15 & $1.0006 \pm 0.0002$ & 1.0000 \\
283.15 & $1.0002 \pm 0.0002$ & 0.9997 \\
288.15 & $0.9994 \pm 0.0002$ & 0.9991 \\
293.15 & $0.9986 \pm 0.0002$ & 0.9982 \\
298.15 & $0.9976 \pm 0.0002$ & 0.9970 \\
303.15 & $0.9962 \pm 0.0001$ & 0.9956 \\
308.15 & $0.9945 \pm 0.0001$ & 0.9940 \\
313.15 & $0.9925 \pm 0.0001$ & 0.9922 \\
318.15 & $0.9904 \pm 0.0001$ & 0.9902 \\
323.15 & $0.9881 \pm 0.0001$ & 0.9880 \\
328.15 & $0.9857 \pm 0.0001$ & 0.9857 \\
333.15 & $0.9830 \pm 0.0001$ & 0.9832 \\
338.15 & $0.9800 \pm 0.0001$ & 0.9806 \\
343.15 & $0.9769 \pm 0.0001$ & 0.9778 \\
348.15 & $0.9737 \pm 0.0001$ & 0.9748 \\
353.15 & $0.9705 \pm 0.0001$ & 0.9718 \\
358.15 & $0.9671 \pm 0.0001$ & 0.9686 \\
363.15 & $0.9636 \pm 0.0001$ & 0.9653 \\
368.15 & $0.9598 \pm 0.0001$ & 0.9619 \\
373.15 & $0.9559 \pm 0.0002$ & 0.9583 \\
\hline & &
\end{tabular}


Table S2. Thermal expansion coefficient (in $10^{-4} / \mathrm{K}$ ) calculated using TIP7P model over the temperatures between $253 \mathrm{~K}$ and $373 \mathrm{~K}$ at 1 atm

\begin{tabular}{lll}
\hline Temperature & TIP7P & Experiment \\
\hline 253.15 & $-5.128 \pm 1.208$ & -6.604 \\
258.15 & $-4.060 \pm 1.208$ & -4.488 \\
263.15 & $-2.695 \pm 0.683$ & -2.911 \\
268.15 & $-1.887 \pm 0.612$ & -1.678 \\
273.15 & $-0.893 \pm 0.518$ & -0.678 \\
277.15 & $-0.016 \pm 0.457$ & 0.003 \\
283.15 & $1.283 \pm 0.398$ & 0.879 \\
288.15 & $1.746 \pm 0.364$ & 1.508 \\
293.15 & $1.750 \pm 0.341$ & 2.068 \\
298.15 & $2.319 \pm 0.317$ & 2.573 \\
303.15 & $3.168 \pm 0.296$ & 3.034 \\
308.15 & $3.758 \pm 0.281$ & 3.459 \\
313.15 & $4.145 \pm 0.260$ & 3.855 \\
318.15 & $4.426 \pm 0.245$ & 4.226 \\
323.15 & $4.730 \pm 0.235$ & 4.578 \\
328.15 & $5.216 \pm 0.223$ & 4.912 \\
333.15 & $5.843 \pm 0.215$ & 5.233 \\
338.15 & $6.307 \pm 0.210$ & 5.541 \\
343.15 & $6.482 \pm 0.208$ & 5.840 \\
348.15 & $6.539 \pm 0.207$ & 6.130 \\
353.15 & $6.736 \pm 0.203$ & 6.414 \\
358.15 & $7.112 \pm 0.200$ & 6.692 \\
363.15 & $7.597 \pm 0.205$ & 6.966 \\
368.15 & $8.113 \pm 0.226$ & 7.237 \\
373.15 & $8.401 \pm 0.266$ & 7.506 \\
\hline & & \\
\hline
\end{tabular}


Table S3. Isothermal compressibility (in 10-6/bar) calculated using TIP7P model over the temperatures between $253 \mathrm{~K}$ and $373 \mathrm{~K}$ at $1 \mathrm{~atm}$

\begin{tabular}{llll}
\hline Temperature & TIP7P & fitted & Experiment \\
\hline 253.15 & $62.210 \pm 2.967$ & 61.951 & 64.451 \\
258.15 & $60.125 \pm 2.000$ & 60.028 & 59.516 \\
263.15 & $57.669 \pm 1.599$ & 58.325 & 55.855 \\
268.15 & $56.834 \pm 1.431$ & 56.832 & 53.061 \\
273.15 & $55.922 \pm 1.236$ & 55.541 & 50.885 \\
277.15 & $54.893 \pm 1.116$ & 54.649 & 49.481 \\
283.15 & $53.060 \pm 0.961$ & 53.534 & 47.808 \\
288.15 & $51.865 \pm 0.871$ & 52.800 & 46.733 \\
293.15 & $51.993 \pm 0.836$ & 52.236 & 45.891 \\
298.15 & $52.607 \pm 0.820$ & 51.832 & 45.246 \\
303.15 & $52.653 \pm 0.798$ & 51.581 & 44.769 \\
308.15 & $52.178 \pm 0.750$ & 51.473 & 44.439 \\
313.15 & $51.818 \pm 0.688$ & 51.501 & 44.238 \\
318.15 & $51.507 \pm 0.652$ & 51.656 & 44.153 \\
323.15 & $51.288 \pm 0.629$ & 51.931 & 44.173 \\
328.15 & $51.355 \pm 0.594$ & 52.316 & 44.291 \\
333.15 & $51.913 \pm 0.570$ & 52.803 & 44.499 \\
338.15 & $53.159 \pm 0.576$ & 53.384 & 44.792 \\
343.15 & $54.606 \pm 0.595$ & 54.051 & 45.167 \\
348.15 & $55.368 \pm 0.600$ & 54.795 & 45.620 \\
353.15 & $55.766 \pm 0.592$ & 55.608 & 46.150 \\
358.15 & $56.563 \pm 0.593$ & 56.482 & 46.755 \\
363.15 & $57.638 \pm 0.619$ & 57.408 & 47.435 \\
368.15 & $58.621 \pm 0.693$ & 58.378 & 48.191 \\
373.15 & $58.868 \pm 0.821$ & 59.383 & 49.023 \\
\hline & & & \\
\hline
\end{tabular}


Table S4. Enthalpy of vaporization (in $\mathrm{kcal} / \mathrm{mol}$ ) calculated using TIP7P model over the temperatures between $253 \mathrm{~K}$ and $373 \mathrm{~K}$ at $1 \mathrm{~atm}$.

\begin{tabular}{llll}
\hline Temperature & TIP7P(raw) & TIP7P & Experiment \\
\hline 253.15 & $12.600 \pm 0.004$ & 11.085 & 10.947 \\
258.15 & $12.516 \pm 0.003$ & 11.014 & 10.902 \\
263.15 & $12.427 \pm 0.002$ & 10.938 & 10.855 \\
268.15 & $12.343 \pm 0.002$ & 10.866 & 10.807 \\
273.15 & $12.263 \pm 0.002$ & 10.799 & 10.758 \\
277.15 & $12.197 \pm 0.002$ & 10.742 & 10.718 \\
283.15 & $12.102 \pm 0.002$ & 10.661 & 10.658 \\
288.15 & $12.024 \pm 0.002$ & 10.594 & 10.607 \\
293.15 & $11.946 \pm 0.004$ & 10.527 & 10.556 \\
298.15 & $11.869 \pm 0.005$ & 10.460 & 10.505 \\
303.15 & $11.793 \pm 0.004$ & 10.394 & 10.454 \\
308.15 & $11.718 \pm 0.003$ & 10.329 & 10.403 \\
313.15 & $11.645 \pm 0.002$ & 10.265 & 10.351 \\
318.15 & $11.573 \pm 0.002$ & 10.202 & 10.300 \\
323.15 & $11.501 \pm 0.004$ & 10.138 & 10.248 \\
328.15 & $11.428 \pm 0.004$ & 10.072 & 10.195 \\
333.15 & $11.355 \pm 0.004$ & 10.007 & 10.143 \\
338.15 & $11.285 \pm 0.003$ & 9.943 & 10.090 \\
343.15 & $11.216 \pm 0.003$ & 9.880 & 10.037 \\
348.15 & $11.147 \pm 0.004$ & 9.817 & 9.984 \\
353.15 & $11.077 \pm 0.004$ & 9.752 & 9.930 \\
358.15 & $11.009 \pm 0.004$ & 9.688 & 9.875 \\
363.15 & $10.941 \pm 0.003$ & 9.624 & 9.820 \\
368.15 & $10.874 \pm 0.003$ & 9.560 & 9.764 \\
373.15 & $10.808 \pm 0.003$ & 9.497 & 9.708 \\
\hline
\end{tabular}


Table S5. Isobaric heat capacity (in $\mathrm{cal} / \mathrm{mol} / \mathrm{K}$ ) calculated using TIP7P model over the temperatures between $253 \mathrm{~K}$ and $373 \mathrm{~K}$ at $1 \mathrm{~atm}$

\begin{tabular}{llll}
\hline Temperature & TIP7P(raw) & TIP7P & Experiment \\
\hline 253.15 & $22.746 \pm 0.870$ & 20.073 & 18.934 \\
258.15 & $23.355 \pm 0.574$ & 20.740 & 18.589 \\
263.15 & $22.771 \pm 0.428$ & 20.213 & 18.379 \\
268.15 & $22.405 \pm 0.357$ & 19.903 & 18.243 \\
273.15 & $21.777 \pm 0.314$ & 19.330 & 18.152 \\
277.15 & $21.548 \pm 0.298$ & 19.144 & 18.101 \\
283.15 & $21.157 \pm 0.281$ & 18.817 & 18.048 \\
288.15 & $21.147 \pm 0.273$ & 18.859 & 18.020 \\
293.15 & $21.261 \pm 0.258$ & 19.025 & 18.001 \\
298.15 & $21.285 \pm 0.239$ & 19.099 & 17.989 \\
303.15 & $20.904 \pm 0.224$ & 18.768 & 17.982 \\
308.15 & $20.738 \pm 0.218$ & 18.651 & 17.980 \\
313.15 & $20.347 \pm 0.216$ & 18.308 & 17.980 \\
318.15 & $20.287 \pm 0.209$ & 18.296 & 17.983 \\
323.15 & $20.467 \pm 0.200$ & 18.523 & 17.988 \\
328.15 & $20.353 \pm 0.192$ & 18.455 & 17.995 \\
333.15 & $20.107 \pm 0.189$ & 18.254 & 18.004 \\
338.15 & $20.110 \pm 0.189$ & 18.302 & 18.014 \\
343.15 & $19.934 \pm 0.190$ & 18.170 & 18.026 \\
348.15 & $19.773 \pm 0.190$ & 18.053 & 18.040 \\
353.15 & $19.762 \pm 0.187$ & 18.084 & 18.055 \\
358.15 & $19.498 \pm 0.185$ & 17.863 & 18.072 \\
363.15 & $19.285 \pm 0.191$ & 17.691 & 18.091 \\
368.15 & $19.349 \pm 0.215$ & 17.796 & 18.112 \\
373.15 & $19.282 \pm 0.261$ & 17.770 & 18.136 \\
\hline & & &
\end{tabular}


Table S6. Static dielectric constant calculated using TIP7P model over the temperatures between $253 \mathrm{~K}$ and $373 \mathrm{~K}$ at $1 \mathrm{~atm}$

\begin{tabular}{lll}
\hline Temperature & TIP7P & Experiment \\
\hline 253.15 & $96.315 \pm 10.259$ & 96.486 \\
258.15 & $94.019 \pm 6.269$ & 94.264 \\
263.15 & $93.867 \pm 4.865$ & 92.098 \\
268.15 & $92.708 \pm 4.468$ & 89.986 \\
273.15 & $88.136 \pm 3.740$ & 87.927 \\
277.15 & $84.646 \pm 3.479$ & 86.318 \\
283.15 & $81.975 \pm 3.310$ & 83.963 \\
288.15 & $80.179 \pm 2.846$ & 82.054 \\
293.15 & $77.756 \pm 2.373$ & 80.192 \\
298.15 & $76.098 \pm 2.026$ & 78.375 \\
303.15 & $76.001 \pm 1.869$ & 76.603 \\
308.15 & $76.311 \pm 1.848$ & 74.873 \\
313.15 & $75.379 \pm 1.753$ & 73.184 \\
318.15 & $72.859 \pm 1.591$ & 71.536 \\
323.15 & $70.234 \pm 1.451$ & 69.927 \\
328.15 & $68.171 \pm 1.321$ & 68.355 \\
333.15 & $66.683 \pm 1.199$ & 66.820 \\
338.15 & $65.653 \pm 1.124$ & 65.320 \\
343.15 & $64.714 \pm 1.083$ & 63.855 \\
348.15 & $63.560 \pm 1.025$ & 62.424 \\
353.15 & $62.147 \pm 0.953$ & 61.024 \\
358.15 & $60.661 \pm 0.900$ & 59.656 \\
363.15 & $59.008 \pm 0.869$ & 58.319 \\
368.15 & $56.963 \pm 0.890$ & 57.011 \\
373.15 & $54.812 \pm 0.993$ & 55.732 \\
\hline & &
\end{tabular}


Table S7. Self-diffusion coefficient (in $10^{-5} \mathrm{~cm}^{2} / \mathrm{s}$ ) calculated using TIP7P model over the temperatures between $253 \mathrm{~K}$ and $373 \mathrm{~K}$ at $1 \mathrm{~atm}$

\begin{tabular}{llll}
\hline Temperature & TIP7P & fitted & Experiment \\
\hline 253.15 & $0.7087 \pm 0.0240$ & 0.7653 & 0.4602 \\
258.15 & $0.7687 \pm 0.0255$ & 0.8504 & 0.5935 \\
263.15 & $0.9656 \pm 0.0067$ & 0.9617 & 0.7443 \\
268.15 & $1.1615 \pm 0.0266$ & 1.0981 & 0.9128 \\
273.15 & $1.2957 \pm 0.0601$ & 1.2587 & 1.0990 \\
277.15 & $1.5403 \pm 0.0196$ & 1.4040 & 1.2608 \\
283.15 & $1.7412 \pm 0.0115$ & 1.6487 & 1.5250 \\
288.15 & $1.9996 \pm 0.1607$ & 1.8762 & 1.7650 \\
293.15 & $1.9697 \pm 0.1878$ & 2.1241 & 2.0231 \\
298.15 & $2.3766 \pm 0.0201$ & 2.3915 & 2.2995 \\
303.15 & $2.5310 \pm 0.3548$ & 2.6774 & 2.5940 \\
308.15 & $2.9276 \pm 0.1124$ & 2.9808 & 2.9069 \\
313.15 & $3.3275 \pm 0.0717$ & 3.3008 & 3.2382 \\
318.15 & $3.5890 \pm 0.3468$ & 3.6364 & 3.5879 \\
323.15 & $3.8763 \pm 0.1630$ & 3.9868 & 3.9561 \\
328.15 & $4.1939 \pm 0.0953$ & 4.3508 & 4.3429 \\
333.15 & $5.3109 \pm 0.4460$ & 4.7277 & 4.7483 \\
338.15 & $4.7731 \pm 0.0613$ & 5.1164 & 5.1724 \\
343.15 & $5.5216 \pm 0.0236$ & 5.5160 & 5.6152 \\
348.15 & $5.9893 \pm 0.2372$ & 5.9256 & 6.0767 \\
353.15 & $6.1317 \pm 0.2093$ & 6.3441 & 6.5570 \\
358.15 & $7.0424 \pm 0.9127$ & 6.7707 & 7.0562 \\
363.15 & $7.1282 \pm 0.2971$ & 7.2043 & 7.5743 \\
368.15 & $8.0678 \pm 0.6063$ & 7.6441 & 8.1113 \\
373.15 & $7.7117 \pm 0.0189$ & 8.0891 & 8.6673 \\
\hline & & &
\end{tabular}


Table S8. Densities in liquid-vapor equilibrium (in $\mathrm{g} / \mathrm{cm}^{3}$ ) calculated using TIP7P model at $1 \mathrm{~atm}$.

\begin{tabular}{lclcc}
\hline \multirow{2}{*}{ Temperature } & \multicolumn{2}{c}{ TIP7P } & \multicolumn{2}{c}{ Experiment } \\
\cline { 2 - 5 } & $\rho_{\text {liquid }}$ & $\rho_{\text {gas }}$ & $\rho_{\text {liquid }}$ & $\rho_{\text {gas }}$ \\
\hline 298 & 0.9969 & 0 & 0.9970 & $0.2288 \mathrm{e}-4$ \\
323 & 0.9857 & $0.1769 \mathrm{e}-4$ & 0.9881 & $0.8257 \mathrm{e}-4$ \\
348 & 0.9709 & $0.6871 \mathrm{e}-4$ & 0.9749 & $0.2408 \mathrm{e}-3$ \\
373 & 0.9534 & $0.2376 \mathrm{e}-3$ & 0.9585 & $0.5951 \mathrm{e}-3$ \\
398 & 0.9342 & $0.4914 \mathrm{e}-3$ & 0.9391 & $0.1293 \mathrm{e}-2$ \\
423 & 0.9097 & $0.1202 \mathrm{e}-2$ & 0.9172 & $0.2538 \mathrm{e}-2$ \\
448 & 0.8833 & $0.2161 \mathrm{e}-2$ & 0.8924 & $0.4602 \mathrm{e}-2$ \\
473 & 0.8534 & $0.3965 \mathrm{e}-2$ & 0.8648 & $0.7837 \mathrm{e}-2$ \\
498 & 0.8187 & $0.5672 \mathrm{e}-2$ & 0.8340 & $0.1272 \mathrm{e}-1$ \\
523 & 0.7782 & $0.1064 \mathrm{e}-1$ & 0.7992 & $0.1991 \mathrm{e}-1$ \\
548 & 0.7319 & $0.1905 \mathrm{e}-1$ & 0.7594 & $0.3044 \mathrm{e}-1$ \\
573 & 0.6809 & $0.3382 \mathrm{e}-1$ & 0.7126 & $0.4606 \mathrm{e}-1$ \\
598 & 0.6086 & $0.7372 \mathrm{e}-1$ & 0.6549 & $0.7032 \mathrm{e}-1$ \\
\hline
\end{tabular}


Table S9. Properties of non-polarizable models at $298 \mathrm{~K}$ and $1 \mathrm{~atm}$.

\begin{tabular}{lccccccc}
\hline \multicolumn{1}{c}{ Model } & $\begin{array}{c}\text { TMD } \\
\mathrm{K}\end{array}$ & $\begin{array}{c}\rho \\
\mathrm{g} / \mathrm{cm}^{3}\end{array}$ & $\begin{array}{c}\Delta H_{\text {vap }} \\
\mathrm{kcal} / \mathrm{mol}\end{array}$ & $\begin{array}{c}\alpha_{p} \\
10^{-4} / \mathrm{K}\end{array}$ & $\begin{array}{c}\kappa_{T} \\
10^{-6} / \mathrm{bar}\end{array}$ & $\begin{array}{c}C_{P} \\
\mathrm{cal} / \mathrm{mol} / \mathrm{K}\end{array}$ & $\begin{array}{c}D_{s} \\
10^{-5} \mathrm{~cm}^{2} / \mathrm{s}\end{array}$ \\
\hline SPC/E $^{\mathrm{a}}$ & 241 & 0.998 & 9.89 & 5.0 & 46.1 & 20.7 & 2.5 \\
$\mathrm{TIP}^{\mathrm{b}} \mathrm{P}$ & $182^{\mathrm{e}}$ & 0.982 & 10.45 & 4.1 & 57.4 & 18.74 & 5.5 \\
TIP4P-Ew $^{\mathrm{c}}$ & $274^{\mathrm{e}}$ & 0.9954 & 10.58 & 3.2 & 48.1 & 19.2 & 2.4 \\
TIP5P-E $^{\mathrm{d}}$ & $277^{\mathrm{e}}$ & 1.000 & 10.38 & 4.9 & 52 & 27 & 2.8 \\
TIP7P & 277 & 0.9976 & 10.99 & 2.319 & 52.61 & 21.29 & 2.4 \\
Expt. & $277^{\mathrm{f}}$ & $0.9970^{\mathrm{f}}$ & $10.52^{\mathrm{g}}$ & $2.572^{\mathrm{f}}$ & $45.247^{\mathrm{f}}$ & $18.002^{\mathrm{g}}$ & $2.3^{\mathrm{h}}$ \\
\hline
\end{tabular}

${ }^{\mathrm{a}} \operatorname{Ref} 1-3 .{ }^{\mathrm{b}} \operatorname{Ref} 4 .{ }^{\mathrm{c}} \operatorname{Ref} 5 .{ }^{\mathrm{d}} \operatorname{Ref} 6 .{ }^{\mathrm{e}} \operatorname{Ref} 2 .{ }^{\mathrm{f}} \operatorname{Ref} 7 .{ }^{\mathrm{g}} \operatorname{Ref} 8 .{ }^{\mathrm{h}} \operatorname{Ref} 9$. 


\section{References}

1. Berendsen, H. J. C.; Grigera, J. R.; Straatsma, T. P. The Missing Term in Effective Pair Potentials. J. Phys. Chem. 1987, 91, 6269-6271.

2. Vega, C.; Abascal, J. L. F. Simulating Water with Rigid Non-Polarizable Models: A General Perspective. Phys. Chem. Chem. Phys. 2011, 13, 19663-19688.

3. Izadi, S.; Anandakrishnan, R.; Onufriev, A. V. Building Water Models: A Different Approach. J. Phys. Chem. Lett. 2014, 5, 3863-3871.

4. Jorgensen, W. L.; Chandrasekhar, J.; Madura, J. D.; Impey, R. W.; Klein, M. L. Comparison of Simple Potential Functions for Simulating Liquid Water. J. Chem. Phys. 1983, 79, 926-935.

5. Horn, H. W.; Swope, W. C.; Pitera, J. W.; Madura, J. D.; Dick, T. J.; Hura, G. L.; Head-Gordon, T. Development of an Improved Four-Site Water Model for Biomolecular Simulations: TIP4P-Ew. J. Chem. Phys. 2004, 120, 9665-9678.

6. Rick, S. W. A Reoptimization of the Five-Site Water Potential (TIP5P) for Use with Ewald Sums. J. Chem. Phys. 2004, 120, 6085-6093.

7. Kell, G. S. Density, Thermal Expansivity, and Compressibility of Liquid Water from $0^{\circ}$ to $150^{\circ} \mathrm{C}$ : Correlations and Tables for Atmospheric Pressure and Saturation Reviewed and Expressed on 1968 Temperature Scale. J. Chem. Eng. Data 1975, 20, 97-105.

8. Wagner, W.; Pruß, A. The IAPWS Formulation 1995 for the Thermodynamic Properties of Ordinary Water Substance for General and Scientific Use. J. Phys. Chem. Ref. Data 2002, 31, 387-535.

9. Krynicki, K.; Green, C. D.; Sawyer, D. W. Pressure and Temperature Dependence of Self-Diffusion in Water. Faraday Discuss. Chem. Soc. 1978, 66, 199-208. 